\title{
Mazabraud's syndrome: a new case and review of the literature
}

\author{
Carmine Zoccali • Giuseppe Teori - Umberto Prencipe • \\ Fabio Erba
}

Received: 4 September 2007 /Revised: 19 September 2007 / Accepted: 21 September 2007 / Published online: 24 January 2008

(C) Springer-Verlag 2007

\begin{abstract}
The association between muscular myxomas and fibrous dysplasia is a rare condition known as Mazabraud's syndrome, as reported by Henschen (Verh Dtsch Ges Pathol 21:93-97, 1926) and Mazabraud A and Girard (Rev Rhum Mal Osteoartic 24(9-10):652-659, 1957). We report a case of a 32-year-old woman with multiple myxomas in her right thigh and monomelic fibrous dysplasia. A review of the international literature referring to 67 cases to date was carried out. The syndrome is characterised by the following features: females are twice as likely to be affected as males; the lower limbs are the most frequently affected, fibrous dysplasia is more common in the femur and the pelvis and myxomas in the quadriceps muscle; myxoma is multiple in more than $70 \%$ of cases. Although there has never been any continuity between tumours and bone lesions, a significant correlation between dysplastic bone and myxoma has been revealed.
\end{abstract}

Résumé L'association entre un myxome musculaire et une dysplasie fibreuse est rare (Mazabraud, 3 à 6). Nous avons

\author{
C. Zoccali $\cdot$ G. Teori $\cdot$ U. Prencipe $\cdot$ F. Erba \\ Oncological Orthopaedics Department, \\ Muscular-Skeletal Tissue Bank, IFO-IRE, \\ Roma, Italy \\ G. Teori \\ e-mail: teori@tiscali.it \\ U. Prencipe \\ e-mail: principe@ifo.it \\ F. Erba \\ e-mail: erba@ifo.it \\ C. Zoccali $(\bowtie)$ \\ IFO-Regina Elena Institute, \\ Via Elio Chianesi 53, \\ Rome, Italy \\ e-mail: carminezoccali@libero.it
}

rapporté le cas d'une femme de 32 ans présentant un myxome musculaire multiple, au niveau de la cuisse droite et une atteinte de dysplasie fibreuse. La revue de la littérature internationale permet de rapporter 67 cas caractérisant une telle symptomatologie. Les femmes sont atteintes deux fois plus fréquemment que les hommes et les membres inférieurs également (la dysplasie fibreuse est fréquente au niveau du fémur et du bassin). Le myxome musculaire étant surtout localisé au niveau du quadriceps. Ces myxomes présentent généralement des lésions multiples dans $70 \%$ des cas. Si on ne peut pas mettre en évidence de continuité entre ces deux tumeurs, leur association existe néanmoins.

\section{Case report}

A 32-year-old Caucasian female presented to the plastic surgery department of a general hospital for the evaluation of a mass present in the medial aspect of the right thigh. The diameter was $8 \mathrm{~cm}$, which had gradually increased in size over the past two years since the patient first noted it. The surgeons decided to excise the lesion which was located in the vastus medialis, of oval shape, dimensions $6.5 \times 4.4 \times 3.5 \mathrm{~cm}$, with a hard-elastic consistency and traslucid with myxoid appearance after removal.

Another smaller lesion was removed from the same area.

Histological examination showed a tissue with spike- and star-shaped cells in an abundant myxoid matrix; no mitotic activity was documented. Immuno-histochemical results were slightly positive for s100 and negative for CD99, EMA, CD56 and CD57. A diagnosis of myxoma was proposed.

Nuclear magnetic resonance (NMR) imaging, performed post-operatively, revealed the presence of multiple lesions in the femur and right tibia, with low signal intensity on T1-weighted images (Fig. 1) and high signal intensity on 
fat-suppression sequence images (Fig. 2). Successive Xrays showed evidence of multiple radiodense lesions with smooth margins in the proximal third of the right tibia and right femur, not affecting cortical bone (Figs. 3 and 4).

Clinical examination revealed no shortening. No endocrine problems were noted but "café au lait" spots were present on the medial aspect of the right upper limb (Fig. 5).

Bone scan showed evidence of increased uptake in the proximal third of the right femur and particularly in the proximal right tibia (Fig. 6).

A diagnosis of Mazabraud's syndrome was made and the patient was included in a follow-up programme for patients with fibrous dysplasia. No surgical operation was performed.

\section{Materials and methods}

Since Henschen [3] first described Mazabraud's syndrome in 1926, 68 cases, including ours, have been listed to date. These were reviewed. Table 1 lists the results.

Two of these cases were removed from the study because we could not find the original articles or information in other reviews $[1,8]$.

To validate age onset, a $t$-test for independent groups was applied and Fisher's test was used to analyse the distribution of fibrous dysplasia lesions.

Tsitouridis et al. [9] described a case where precocious puberty was considered at 11 years, but in our review, it was considered normal.

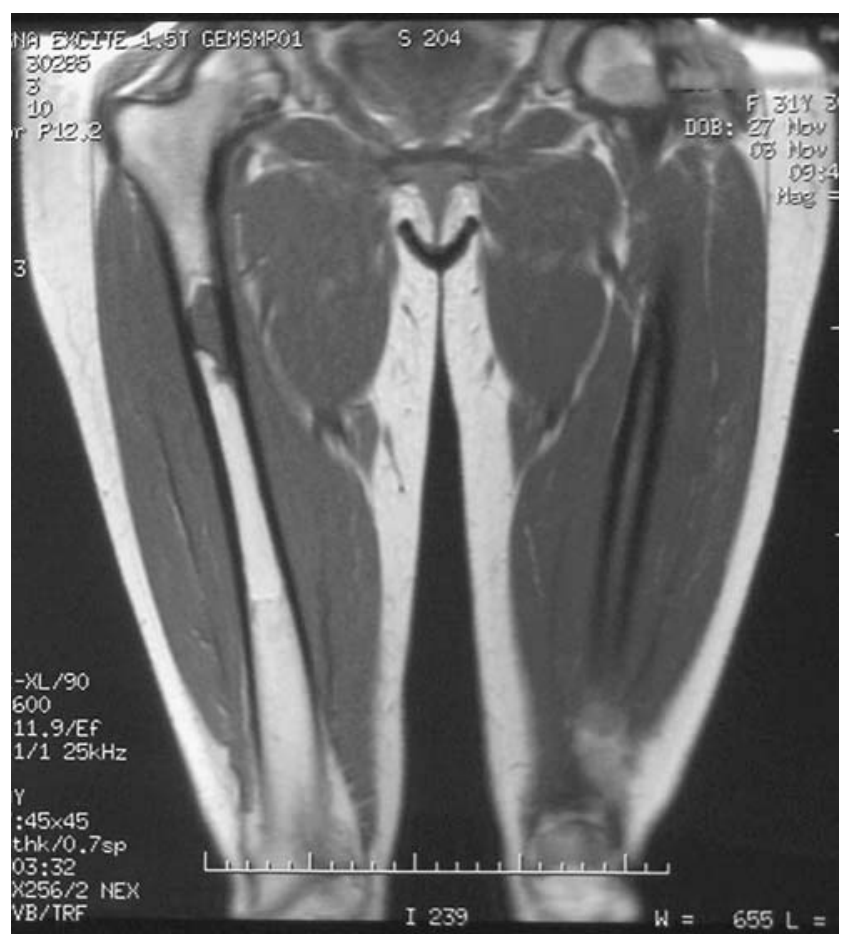

Fig. 1 Low signal intensity on T1-weighted images

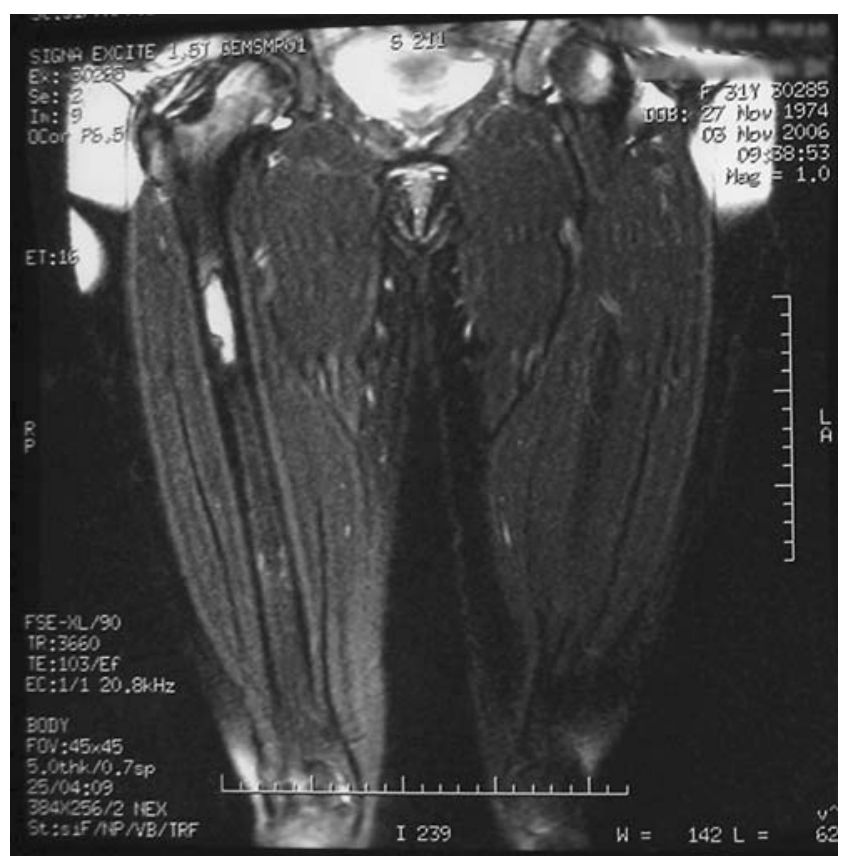

Fig. 2 High signal intensity on fat-suppression sequence images

The syndrome population is composed of 46 women $(67.65 \%)$ and 20 men $(32.35 \%)$.

\section{Results and discussion}

Fibrous dysplasia is a non-malignant infrequent condition caused by a mutation in the GNAS gene, located on chromosome 20q13.2-q13.3, encoded to a $\operatorname{Gs}(\alpha)$ protein and enrolled in proliferation cell activity. Mutations

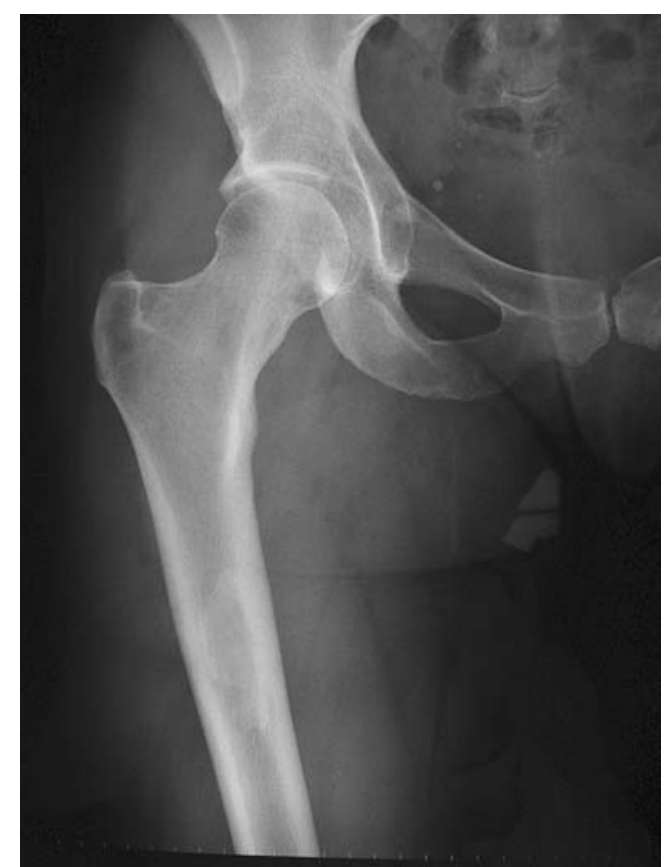

Fig. 3 Proximal right femur; radiodense lesion with smooth margins 


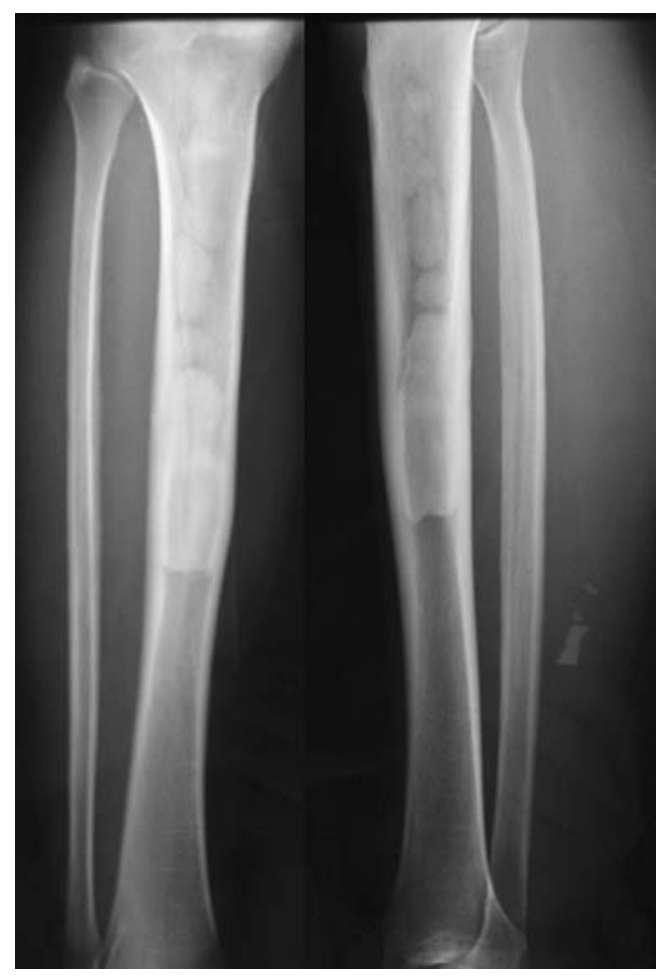

Fig. 4 Right tibia; multiple radiodense lesions with smooth margins

increase protein activity and abnormal proliferation. Mosaic cell distribution is present, leading to a pathological bone when the mutation is in bone cells and to McCune-Albright syndrome when endocrine cells are affected $[2,10]$.

Fibrous dysplasia is characterised by the presence of non-ossifying areas in bone. It may be monostotic or polyostotic and is usually unilateral. It can be associated with endocrine disturbance such as precocious sexual development or goitre and diabetes mellitus. There may be "café au lait spots" indicating a condition known as McCune-Albright syndrome. When associated with myxoma, it is known as Mazabraud's syndrome.

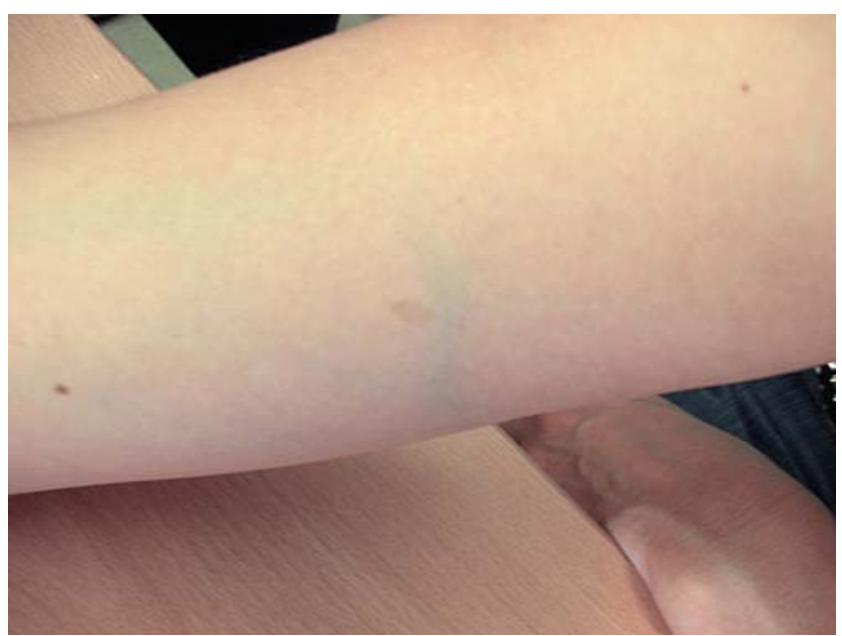

Fig. 5 "Café au lait" spots on the medial aspect of the right arm

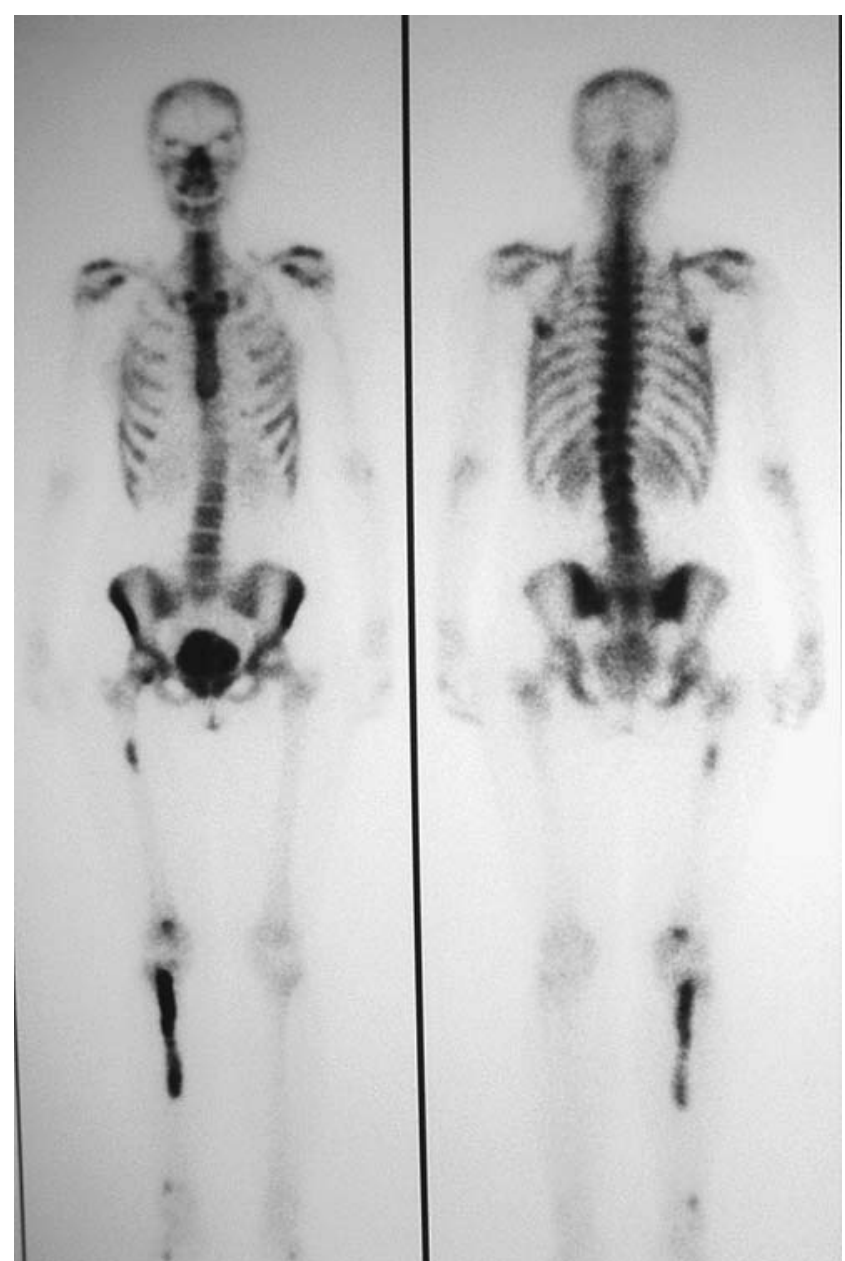

Fig. 6 Increased uptake in the proximal third of the right femur and right tibia

Myxoma is a benign neoplasm usually diagnosed in the sixth to seventh decades of life. It can be single or multiple. In Mazabraud's syndrome, it is frequently multiple and combined with a dysplastic bone without any continuity between the lesions.

The most important feature is that the myxoma develops 12 years before fibrous dysplasia diagnosis. Females are affected twice as often as males. Fibrous dysplasia is often polyostotic and the femur is the most commonly affected. Myxomas are often multiple and thigh muscles are more frequently involved.

Age, sex and race We knew the onset of fibrous dysplasia in 52 cases. It was usually diagnosed between 1 year to 67 years of age, with an average of 32.42 years (variance 315.03 , median 34). In four other case studies, we only knew that it was diagnosed in infancy and in four other cases, diagnosis was achieved before myxoma.

In five cases, no information about the onset of the different components are reported. 
Table 1 Mazabraud's syndrome cases; the black boxes indicate that further explanation is possible or necessary

\begin{tabular}{|c|c|c|c|c|c|c|c|c|c|c|c|c|c|}
\hline \multirow{2}{*}{$\begin{array}{l}\text { Authors } \\
\text { Henschen }\end{array}$} & \multirow{2}{*}{\begin{tabular}{|l|} 
Year \\
1926 \\
\end{tabular}} & \multirow{2}{*}{$\begin{array}{l}\text { Sex } \\
f \\
\end{array}$} & \multirow{2}{*}{$\begin{array}{c}\text { Age at FD } \\
\text { diagnosis } \\
\text { child } \\
\end{array}$} & \multirow{2}{*}{$\begin{array}{c}\text { Age at I } \\
\text { Myxoma } \\
\text { diag. }\end{array}$} & \multicolumn{4}{|c|}{ Fibrous Dysplasia } & \multicolumn{3}{|r|}{ Myxoma } & \multirow{2}{*}{$\begin{array}{c}\begin{array}{c}\text { Café au lait } \\
\text { spots }\end{array} \\
\text { no }\end{array}$} & \multirow{2}{*}{ Associations } \\
\hline & & & & & Mo & UR & & $\operatorname{tg}$ & $\mathrm{M}$ & UR & $\operatorname{tg}$ & & \\
\hline Krogius & 1929 & f & 6 & 26 & Po & B & & $\begin{array}{l}\text { R \& L pe, R \& L } \\
\text { fe, R ti, R fi, } \\
R \& L \text { hu } \\
\end{array}$ & $\mathrm{M}$ & UR & tg, sh, hu & no & Ovarian struma \\
\hline Uehlinger & 1940 & $m$ & 11 & 67 & Po & $B$ & & n.a. & $S$ & & $R$ tg & & \\
\hline Braunwarth & 1953 & $f$ & $\mathrm{nr}$ & 55 & Mo & U L & & n.a. & $\mathrm{S}$ & & $\mathrm{L}$ & & \\
\hline $\begin{array}{l}\text { Mazabraud } \\
\text { and Girard }\end{array}$ & 1957 & $m$ & 22 & 54 & Po & UR & $\mathrm{Em}$ & $\begin{array}{l}\text { hu, sc, fe, pe, II } \\
\text { mt \& falangis }\end{array}$ & $\mathrm{M}$ & U R & $\begin{array}{l}\text { sh (pectoralis } \\
\text { minor) }\end{array}$ & no & no \\
\hline $\begin{array}{l}\text { Heinemann } \\
\text { and Woerth }\end{array}$ & 1958 & $f$ & 18 & 82 & Po & U R & $\mathrm{Mm}$ & n.a. & $\mathrm{M}$ & U R & n.a. & $\mathrm{R}$ & gouter \\
\hline Laporte et al. & 1961 & $f$ & 12 & 24 & Po & U R & Em & n.a. & $\mathrm{S}$ & & $\mathrm{R}$ & $\mathrm{R}$ & $\begin{array}{l}\text { precocious puberty, } \\
\text { hyperthyroidisme }\end{array}$ \\
\hline Lick & 1962 & $m$ & 18 & 59 & Po & $B$ & & n.a. & M & U R & n.a. & & gouter \\
\hline $\begin{array}{l}\text { Mazabraud } \\
\text { et al. }\end{array}$ & 1967 & $f$ & infant & $\mathrm{nr}$ & Po & UL & $\mathrm{Em}$ & n.a. & $\mathrm{M}$ & U R & n.a. & $\mathrm{R}$ & $\begin{array}{l}\text { precocious puberty, } \\
\text { osteosarcoma }\end{array}$ \\
\hline Roze et al. & 1967 & $f$ & 20 & 38 & Po & B & & $\begin{array}{l}R \& L \text { pe, } R \& L \\
\text { fe, } R \text { \& } L \text { ti, } R \text { \& } L \\
\text { fi, } R \text { \& } L \text { hu, } R \text { ra, } \\
\text { R carpus }\end{array}$ & $\mathrm{M}$ & U R & $\begin{array}{l}\text { pe, leg, sh, } \\
\text { dorsus }\end{array}$ & $\begin{array}{l}\text { R abdomen } \\
\& s c\end{array}$ & $\begin{array}{l}\text { precocious puberty, uterin } \\
\text { fibroma }\end{array}$ \\
\hline Semat & 1969 & $m$ & 30 & 47 & Po & $\mathrm{B}$ & & $\begin{array}{l}R \& L \text { pe, } L \text { fe, } R \\
\& L \text { ti, } \\
L-L 4 \text { trasversal } \\
\text { processus }\end{array}$ & $\mathrm{S}$ & & $L \operatorname{tg}$ & no & thyroid adenoma, pagetoid sk \\
\hline Wirth & 1971 & $m$ & 3 & 17 & Po & $\mathrm{B}$ & & $\begin{array}{l}\text { R \& } \mathrm{L} \text { fe, } \mathrm{R} \text { e } \mathrm{L} \\
\text { hu, ti, ul, rb, ph, } \\
\text { mtt bones }\end{array}$ & $\mathrm{M}$ & $U \mathrm{UL}$ & arm, thorax & L sh & no \\
\hline Wirth & 1971 & $m$ & 18 & 33 & Po & $\mathrm{B}$ & & $\begin{array}{l}\text { sk, rb, R \& L pe, } \\
\text { L fe, L hu }\end{array}$ & $\mathrm{M}$ & U R & tg, arm & R head & pagetoid sk \\
\hline Lejeune et al. & 1972 & $m$ & 41 & 39 & Po & $\mathrm{B}$ & & $\begin{array}{l}\mathrm{L} \text { fe, } \mathrm{L} \mathrm{sc}, \mathrm{R} \text { hu, } \mathrm{R} \\
\text { ra, R mt bones }\end{array}$ & $\mathrm{M}$ & U R & pe, sh, arm & L gluteus & no \\
\hline Ireland et al. & 1973 & $f$ & 49 & 49 & Mo & $U \mathrm{~L}$ & & fe & $\mathrm{M}$ & B & $\begin{array}{l}\text { R pe, } L \text { tg, R \& } \\
\text { L arm }\end{array}$ & L arm, L h\& & no \\
\hline Ireland et al. & 1973 & $f$ & 59 & 52 & Po & U R & $\mathrm{Mm}$ & pe, fe & $\mathrm{M}$ & U R & $\mathrm{R}$ pe, $\mathrm{R}$ tg & no & abdominal tumors \\
\hline Ireland et al. & 1973 & $f$ & 23 & 28 & Po & U L & $\mathrm{Mm}$ & fe, ti & $\mathrm{M}$ & UL & pe, $\operatorname{tg}$ & no & brest fibrosarcoma \\
\hline Logel & 1976 & $f$ & child & 41 & Po & B & & $\begin{array}{l}\text { sk, R md, R pe, R } \\
\text { fe }\end{array}$ & $M$ & B & $\begin{array}{l}\text { R sk, L md, R } \\
\text { tg, }\end{array}$ & $\begin{array}{l}\text { neck, sacral } \\
\text { bone }\end{array}$ & $\begin{array}{l}\text { precocious puberty, diabetes } \\
\text { mellitus, gouter, pagetoidsk, } \\
\text { short stature, uterinus } \\
\text { fibroadenoma }\end{array}$ \\
\hline Berkhoff & 1981 & & & & & & & & & & & & \\
\hline Sedmak et al. & 1983 & $m$ & 33 & 50 & Po & U L & $\mathrm{Mm}$ & fe, ti & $\mathrm{S}$ & & $L \operatorname{tg}$ & no & lipoma (?) \\
\hline $\begin{array}{l}\text { Lever and } \\
\text { Pettingale }\end{array}$ & 1983 & $f$ & 1 & 50 & Po & $B$ & & $\mathrm{R} \& \mathrm{~L}$ pe, $\mathrm{L} \mathrm{cl}, \mathrm{rb}$ & $\mathrm{S}$ & & $R$ tg & no & $\begin{array}{l}\text { precocious puberty, short } \\
\text { stature, osteomalacia }\end{array}$ \\
\hline $\begin{array}{l}\text { Segev and } \\
\text { Reiner }\end{array}$ & 1985 & & & & & & & & & & & & \\
\hline Witkin et al. & 1986 & $m$ & 15 & 40 & Po & B & & $\begin{array}{l}R \& \text { \& fe, } L \text { ti, } R \text { \& } \\
L \text { fi }\end{array}$ & $\mathrm{S}$ & & $L \operatorname{tg}$ & no & $\begin{array}{l}\text { Osteogenic sarcoma L ti, } \\
\text { emoglobin J Baltimora e S }\end{array}$ \\
\hline Blasier et al. & 1986 & $f$ & 57 & 55 & Po & U L & Em & fe, hu, ra & $\mathrm{M}$ & $U \mathrm{~L}$ & thorax, tg & no & no \\
\hline Biagini et al. & 1987 & $f$ & 10 & 42 & Po & B & & $\begin{array}{l}\text { L pe, } R \text { \& } L \text { fe, } L \\
\text { ti, L fi, L hu }\end{array}$ & $M$ & B & $\begin{array}{l}R \& \text { \& } L e, R \& L \\
\operatorname{tg}, L \text { sh }\end{array}$ & no & no \\
\hline $\begin{array}{l}\text { Glass-Royal } \\
\text { et al. }\end{array}$ & 1989 & $\mathrm{~m}$ & 33 & 33 & Po & B & & sk, L hu, R \& L h\& & S & & L arm & $\begin{array}{l}\text { B dorsus, } \\
\text { shs, limbs }\end{array}$ & facial asymmetry \\
\hline $\begin{array}{l}\text { Sundaram } \\
\text { et al. }\end{array}$ & 1989 & $f$ & 31 & 31 & Mo & U R & & fe & $\mathrm{S}$ & & $R$ tg & no & no \\
\hline $\begin{array}{l}\text { Gianoutsos } \\
\text { et al. }\end{array}$ & 1990 & $m$ & 53 & 39 & Mo & UR & & fe & $\mathrm{M}$ & U R & $\operatorname{tg}$ & no & no \\
\hline Prayson & 1992 & $f$ & 5 & 36 & Po & U R & & pe, fe, rb & $\mathrm{S}$ & & $\mathrm{R} \operatorname{tg}$ & back & R carpal tunner syndrome \\
\hline $\begin{array}{l}\text { Gober and } \\
\text { Nicholas }\end{array}$ & 1993 & $f$ & 32 & 37 & Mo & $U \mathrm{~L}$ & & fe & $\mathrm{M}$ & $U \mathrm{~L}$ & $\operatorname{tg}, \mathrm{pe}$ & no & no \\
\hline Aoki et al. & 1995 & $f$ & 37 & 67 & Po & $\mathrm{B}$ & & $\begin{array}{l}R \& \text { \& pe, R \& L } \\
\text { fe, }\end{array}$ & $\mathrm{M}$ & B & $R \& L$ th & no & no \\
\hline Aoki et al. & 1995 & $f$ & 46 & 46 & Po & U R & $\mathrm{Mm}$ & pe, fe & $\mathrm{S}$ & & $\mathrm{R} \operatorname{tg}$ & no & no \\
\hline Fujii et al. & 1996 & $f$ & 44 & 47 & Po & U R & Em & rb, ti & $\mathrm{M}$ & $\mathrm{B}$ & $R \& L$ pe, $R$ tg & no & no \\
\hline Limouzy et al. & 1996 & $\mathrm{~m}$ & 40 & 45 & Mo & U R & $\mathrm{Mm}$ & pe & $S$ & & R pe & no & no \\
\hline $\begin{array}{l}\text { Court-Payen } \\
\text { at al }\end{array}$ & 1997 & $f$ & 50 & 40 & Po & U R & Em & sk, rb & $\mathrm{M}$ & UR & Thoracis wall & no & no \\
\hline Szendroi et al. & 1998 & $f$ & 17 & 52 & Po & $B$ & & $\begin{array}{l}\text { R \& L pe, R \& L ti, } \\
\text { L fi, L I mtt bone }\end{array}$ & $\mathrm{M}$ & $B$ & $\begin{array}{l}R \& L p e, R \& L \\
\operatorname{tg}\end{array}$ & no & multinodular gouter \\
\hline Szendroi et al. & 1998 & $f$ & 34 & 27 & Po & B & & $\begin{array}{l}\text { R \& } L \text { pe, R \& L } \\
\text { fe, R ti, R fi }\end{array}$ & $\mathrm{M}$ & B & $\begin{array}{l}\text { R \& L pe, R \& L } \\
\operatorname{tg}\end{array}$ & no & no \\
\hline Szendroi et al. & 1998 & $f$ & 38 & 54 & Po & U L & Em & pe, fe, fi, & $\mathrm{S}$ & & $\mathrm{Ltg}$ & L & no \\
\hline
\end{tabular}


Table 1 (continued)

\begin{tabular}{|c|c|c|c|c|c|c|c|c|c|c|c|c|c|}
\hline Cabral et al. & 1998 & $\mathrm{~m}$ & 44 & 29 & Po & UR & $\mathrm{Em}$ & sk, pe, fe, ti, rb & $M$ & U R & $\begin{array}{l}\text { tg, } \\
\text { supraclavicular } \\
\text { fossa }\end{array}$ & no & no \\
\hline $\begin{array}{l}\text { Lopez-Ben } \\
\text { et al. }\end{array}$ & 1999 & $f$ & 5 & 40 & Po & B & & $\begin{array}{l}R \text { \& \& L pe, R \& L } \\
\text { fe, L ra, sk }\end{array}$ & $M$ & B & R forarm, L pe & $\begin{array}{l}\mathrm{L} \\
\text { predominant }\end{array}$ & $\begin{array}{l}\text { precocious puberty, } \\
\text { osteosarcoma R ra }\end{array}$ \\
\hline $\begin{array}{l}\text { Thomacot } \\
\text { et al. }\end{array}$ & 1999 & $f$ & 36 & 36 & Po & U L & $\mathrm{Mm}$ & fe, ti & $S$ & & $L \operatorname{tg}$ & yes & \\
\hline Walker et al. & 1999 & $\mathrm{~m}$ & 67 & 67 & Mo & UR & & fe & $M$ & U R & pe, tg, leg & n.r. & no \\
\hline $\begin{array}{l}\text { Kransdorf } \\
\text { et Murphey }\end{array}$ & 1999 & $f$ & 34 & 34 & Po & UR & $\mathrm{Mm}$ & ti, fi & S & & R leg & no & no \\
\hline Okamoto et al. & 2000 & $f$ & 17 & 52 & Po & B & n.r. & n.r. & $M$ & B & n.r. & n.r. & multinodular gouter \\
\hline Okamoto et al. & 2000 & $f$ & 34 & 27 & Po & $\mathrm{B}$ & n.r. & fe, pe & $M$ & B & pe, $R \&$ \& tg & n.r. & n.r. \\
\hline Okamoto et al. & 2000 & $f$ & 38 & 55 & Po & UL & Em & n.r. & $\mathrm{S}$ & & $\mathrm{Ltg}$ & yes & n.r. \\
\hline Struk et al. & 2000 & $f$ & 49 & 53 & Mo & U R & & fe & S & & $\mathrm{R}$ tg & no & no \\
\hline Faivre et al. & 2001 & $f$ & 35 & 35 & Po & $B$ & & $\begin{array}{l}\text { L pe, R \& L fe, L } \\
\text { ti, L fi }\end{array}$ & $M$ & B & $\begin{array}{l}R \& L \text { tg, L leg, } \\
\text { L arm }\end{array}$ & yes & $\begin{array}{l}\text { breast fibrous dysplasia, } \\
\text { multinodular gouter }\end{array}$ \\
\hline Faivre et al. & 2001 & $\mathrm{~m}$ & 18 & 42 & Po & B & & R fe, L hu & $M$ & B & $\mathrm{R}$ tg, L leg & no & no \\
\hline $\begin{array}{l}\text { Delabrousse } \\
\text { et al. }\end{array}$ & 2001 & $f$ & 40 & 40 & Mo & U R & & fe & $\mathrm{M}$ & UR & pe, $\operatorname{tg}$ & no & no \\
\hline Pollandt et al. & 2002 & $f$ & before & 42 & Po & $U \mathrm{~L}$ & $\mathrm{Mm}$ & pe, fe & $M$ & UR & pe & no & no \\
\hline Iwasko et al. & 2002 & $f$ & 39 & 24 & Po & $U \mathrm{~L}$ & $\mathrm{Em}$ & $\mathrm{pe}, \mathrm{fe}, \mathrm{ti}$ & $\mathrm{M}$ & $U \mathrm{~L}$ & $\mathrm{Ltg}$ & no & n.r. \\
\hline Iwasko et al. & 2002 & $f$ & before & 39 & Po & $B$ & & sk, L pe, R fe & $M$ & $B$ & $\operatorname{tg} R$ e L & $\begin{array}{l}\text { R neck, } R \\
\text { back }\end{array}$ & no \\
\hline Iwasko et al. & 2002 & $f$ & before & 63 & Po & UR & Em & pe, fe, ti & $\mathrm{S}$ & & $L \operatorname{tg}$ & no & no \\
\hline Iwasko et al. & 2002 & $f$ & $52 ?$ & $52 ?$ & Po & U R & $\mathrm{Em}$ & $\mathrm{rb}, \mathrm{pe}$ & $M$ & U R & pe & n.r. & n.r. \\
\hline Iwasko et al. & 2002 & $\mathrm{~m}$ & $49 ?$ & $49 ?$ & Mo & U L & & fe & $\mathrm{M}$ & UL & pe, tg, leg & n.r. & n.r. \\
\hline Iwasko et al. & 2002 & $f$ & $65 ?$ & $65 ?$ & Po & UR & $\mathrm{Mm}$ & h\& & $\mathrm{S}$ & & $\mathrm{R} \mathrm{h \&}$ & n.r. & n.r. \\
\hline Iwasko et al. & 2002 & $f$ & $56 ?$ & $56 ?$ & Po & U R & $\mathrm{Mm}$ & ra, ul & $\mathrm{S}$ & & $\mathrm{R}$ forarm & n.r. & n.r. \\
\hline $\begin{array}{l}\text { Tsitouridis } \\
\text { et al. }\end{array}$ & 2002 & $f$ & 5 & 28 & Po & $\mathrm{B}$ & & $\begin{array}{l}\text { sk, } R \& L \text { pe, } R \& \text { } \\
L \text { fe, } R \& L \text { ti, rb, } \\
R \& L \text { hu }\end{array}$ & $S$ & & $\mathrm{R} \operatorname{tg}$ & $\begin{array}{l}\text { face, neck, } \\
\text { back }\end{array}$ & precocious puberty (5y) \\
\hline Fang et al. & 2003 & $\mathrm{~m}$ & child & 33 & Po & UR & Em & fe, $\mathrm{ti}$ & $\mathrm{S}$ & & R leg & no & no \\
\hline Fertikh & 2003 & $f$ & 59 & 59 & Po & B & & n.r. & $M$ & UL & L pe & yes & $\begin{array}{l}\text { precocious puberty, } \\
\text { hypothyroidism }\end{array}$ \\
\hline Jhala et al. & 2003 & $f$ & $44 ?$ & $44 ?$ & Po & U R & $\mathrm{Mm}$ & hu, ra & $\mathrm{M}$ & n.r. & $\mathrm{R}$ arm & yes & osteosarcoma \\
\hline $\begin{array}{l}\text { Kabukcuoglu } \\
\text { et al. }\end{array}$ & 2004 & $f$ & 52 & 52 & Po & $U \mathrm{~L}$ & $\mathrm{Em}$ & fe, ti & S & & $\mathrm{Ltg}$ & no & no \\
\hline $\begin{array}{l}\text { Muthukumar } \\
\text { et al. }\end{array}$ & 2005 & $\mathrm{~m}$ & before & 37 & Po & U R & $\mathrm{Mm}$ & hu, ra & $M$ & U R & arm & no & no \\
\hline $\begin{array}{l}\text { Nguyen and } \\
\text { Ram }\end{array}$ & 2005 & $\mathrm{~m}$ & 48 & 48 & Po & U R & $\mathrm{Mm}$ & $\mathrm{cl}, \mathrm{hu}, \mathrm{ra}$ & $M$ & U R & arm & no & no \\
\hline Endo et al. & 2006 & $\mathrm{~m}$ & 64 & 64 & Mo & U R & & fe & $S$ & & $\mathrm{R} \operatorname{tg}$ & no & no \\
\hline Endo et al. & 2006 & $f$ & 64 & 60 & Mo & UR & & fe & $\mathrm{S}$ & & $R$ tg & no & no \\
\hline Present papier & 2007 & $f$ & 32 & 32 & Po & U R & $\mathrm{Mm}$ & fe, ti & $\mathrm{S}$ & & $R$ tg & $\mathrm{R}$ arm & no \\
\hline
\end{tabular}

Abbrevation R: right, L: left, Mo: monostotic, Po: polyostotic, U: unilateral, B: bilateral, S: single, M: multiple, Em: emisomic, Mm: monomelic, sk: skull, md: mandibola, pe: pelvis, fe: femur, ti: tibia, fi: fibula, metatarsus: mtt, cl: clavicole, sc: scapula, hu: humerus, ra: radius, ul: ulna, mt: metacarpus, ph: phalanges, rb: ribs, tg: tigh, sh: shoulder, n.a.:not available, n.r.:not reported

In one case, the age at diagnosis was not available.

In 60 cases, we knew the age of myxoma onset, which usually arose between 17 to 82 years of age, with an average of 44.32 years (variance 177.27, median 42).

As already mentioned, in five cases, age was not recorded at diagnosis of different syndrome components, whereas in one case, there is no information about myxoma age onset.

In 14 cases, fibrous dysplasia had been discovered with myxoma onset; in nine cases, it was noted after and in 29 cases, it was noted before myxoma. On average, myxoma becomes significantly symptomatic 12.06 years after fibrous dysplasia (data from 52 cases, variance 274.92, $p<0.001$ ).

Although fibrous dysplasia and myxomas, when isolated, have no sex-related incidence, when associated, women revealed a two-fold greater incidence than men (46 females and 20 males).

Although a major incidence in Caucasian people are reported, it is not possible to make any conclusion about race predominance because of the rarity of the syndrome and the high rate of misdiagnosis.

Localisation; in only one case did we have information about lesion localisation In 13 cases, fibrous dysplasia was monostotic (19.70\%); in 53 cases, it was polyostotic (80.30\%). In 24 cases, fibrous dysplasia was bilateral and in 29 cases, it was unilateral. Unilateral forms were distinguished as follows: 18 right, 10 left and one not specified; 15 were hemimelic and 14 were monostotic. 
Despite the fact that polyostotic fibrous dysplasia is more frequent in females than in males this difference has a low score of significance (Fischer test, $p=0.51$ ), therefore, the distribution is not sex-related.

The distribution of the fibrous dysplasia was recorded in 57 patients and was found to be most frequently located in the femur (47 in the femur, ten only in the femur and 25 in the pelvis of which six were bilateral). Precise fibrous dysplasia distribution was noted in 57 patients and we found that the femur was the more frequent localisation (47 have femur localisation cases and 10 had localisation only following pelvis localisation in 25 cases, six of them bilateral).

Overall, of the 66 patients for whom data was available, $26(39.39 \%)$ cases presented with a single myxoma and the remaining 40 cases presented multiple myxomas; 13 of them were bilateral, one was not specificated and 26 were unilateral (73.08\% right and $26.92 \%$ left).

In 60 patients, we knew the exact site; the most frequent localisation was the thigh ( 40 cases), followed by the pelvis with 17 cases.

In 13 cases, the upper arm was involved and in six of these it was the sole site.

Signs and symptoms The following observations are based on 45 cases where clinical information was available.

Myxomas can be painful or asymptomatic and noted only for increasing size. Myxoma can limit movement if located near a joint. Fibrous dysplasia was also asymptomatic nevertheless, in 10 cases, there was a history of pathological fractures most of them multiple. In 25 cases, there was associated McCune-Albright syndrome (five cases with only endocrine disturbances, 11 with "café au lait" spots and nine with both). The most frequent endocrine pathologies were thyroid problems and precocious puberty; one case of diabetes and three cases of Paget's disease of the skull were also reported. Four cases of malignant change in FD are described in the literature, and it may be that malignant change is more common in Mazabraud's syndrome $[4,5,7,11]$.

\section{Conclusion}

Although the association between fibrous dysplasia and myxomas is rare, it is probably underestimated because of frequent misdiagnosis. Females are twice as likely to be affected than males.

Fibrous dysplasia is usually diagnosed before or at the same time as the myxoma, following routine X-ray or magnetic resonance imaging.

Symptoms are related to the two different components; asymptomatic cases are common, so that pathological fractures and increasing size of painful or painless masses, may be present at the onset.

Endocrine disturbances, such as thyroid pathologies, growth disturbance and precocious sexual development, or "café au lait" spots can be associated in the McCuneAlbright syndrome. The lower limbs are the most frequently affected. Fibrous dysplasia is more common in the femur and the pelvis, and myxomas in the quadriceps muscle. Nevertheless, all other sites are possible. When a myxoma is identified, careful examination may reveal further tumours since in over $70 \%$ they are multiple. In no case has there been continuity between tumours and bone lesions, although in most of them, there is a significant correlation between dysplastic bone and myxoma. Actually, although several hypotheses have been proposed, the aetiology of Mazabraud syndrome is still unclear.

The therapy for the myxoma is excision; fibrous dysplasia is only followed-up because there have been four cases of malignant change reported so that a high level of suspicion is mandatory.

\section{References}

1. Berkhoff WB, ter Bruggen Hugenholtz FI, Ingenhoes R (1981) A patient with multiple intramuscular myxomas as an extra-osseous manifestation of fibrous dysplasia. Ned Tijdschr Geneeskd 125 (36):1460-1462

2. de Sanctis L, Delmastro L, Russo MC, Matarazzo P, Lala R, de Sanctis C (2006) Genetics of McCune-Albright syndrome. J Pediatr Endocrinol Metab 19(Suppl 2):577-582

3. Henschen F (1926) Fall von ostitis fibrosa mit multiplen tumoren in der umgebenden muskuratur. Verh Dtsch Ges Pathol 21:93-97

4. Jhala DN, Eltoum I, Carroll AJ, Lopez-Ben R, Lopez-Terrada D, Rao PH, Pettenati MJ, Siegal GP (2003) Osteosarcoma in a patient with McCune-Albright syndrome and Mazabraud's syndrome: a case report emphasizing the cytological and cytogenetic findings. Hum Pathol 34(12):1354-1357

5. Lopez-Ben R, Pitt MJ, Jaffe KA, Siegal GP (1999) Osteosarcoma in a patient with McCune-Albright syndrome and Mazabraud's syndrome. Skeletal Radiol 28(9):522-526

6. Mazabraud A, Girard J (1957) A peculiar case of fibrous dysplasia with osseous and tendinous localizations. Rev Rhum Mal Osteoartic 24(9-10):652-659

7. Mazabraud A, Semat P, Roze R (1967) Apropos of the association of fibromyxomas of the soft tissues with fibrous dysplasia of the bones. Presse Med 75(44):2223-2228

8. Segev Z, Reiner S (1985) Intramuscular myxoma associated with fibrous dysplasia of adjacent bone. Harefuah 108(10):493-494

9. Tsitouridis I, Sayegh FE, Natsis K, Goutsaridou F, Melidis D, Emmanouilidou M (2003) Mazabraud's syndrome: MRI evaluation. Eur J Radiol Extra 45(2):83-87(5)

10. Weinstein LS (2006) G(s)alpha mutations in fibrous dysplasia and McCune-Albright syndrome. J Bone Miner Res 21(Suppl 2): P120-P124

11. Witkin GB, Guilford WB, Siegal GP (1986) Osteogenic sarcoma and soft tissue myxoma in a patient with fibrous dysplasia and hemoglobins JBaltimore and S. Clin Orthop Relat Res 204:245-252 